\title{
Self-reported disability in women with fibromyalgia from a tertiary care center
}

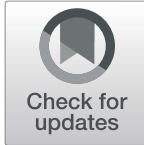

Gabriel Horta-Baas ${ }^{1 *}$ (D) and Maria del Socorro Romero-Figueroa ${ }^{2}$ (D)

\begin{abstract}
Background: The World Health Organization Disability Assessment Schedule (WHODAS) 2.0 is a generic instrument to assess disability. Pain and psychological factors seem to play a pronounced disabling role in fibromyalgia (FM). There are few studies that investigate the factors associated with disability in patients with fibromyalgia from the patient's perspective. Information about FM disability using self-reported questionnaires is limited. This study aimed to assess the relationship between the ordinal response variable (degree of disability), and four explanatory variables: pain intensity, depression, anxiety, and alexithymia.

Methods: One hundred fifteen women with FM were enrolled in the cross-sectional study. For the assessment of disability the WHODAS 2.0 (36-item version) was used. Univariate and multivariate (ordinal logistic regression) analyses were performed to assess the relationship between pain (Visual Analogue Scale), depression and anxiety (Hospital Anxiety and Depression Scale), alexithymia (Modified Toronto Alexithymia Scale) and disability.

Results: Disability was detected by global WHODAS score in 114 patients (99\%), with the corresponding percentages for mild, moderate and severe disability being 11.3, 46.96 and 40.87\%, respectively. Global WHODAS score was more severe among subjects with depression (50 vs 36.4, $p<0.001$, effect size $=0.33$ ) and alexithymia (50 vs 33.6, $p<0.001$, effect size $=0.38$ ). Pain intensity mean scores for mild, moderate and severe disability were 5.0, 6.1 and 7.3 , respectively $(p<0.001$, omega-squared $=0.12)$. Pain intensity explained the global disability degree and its domains except for the cognitive one. Whereas, depression explained cognitive and personal relation domains. On the other hand, alexithymia explained global disability degree and all domains of WHODAS 2.0 questionnaire.
\end{abstract}

Conclusions: Most of the patients with fibromyalgia perceived themselves with moderate to severe disability. The main explanatory variables of the perceived disability were the pain intensity and psychological factors (alexithymia and depression).

Keywords: Fibromyalgia, Chronic pain, Mood disorders, Affective symptoms, Disabled persons, Disability evaluation, WHODAS

\section{Introduction}

Fibromyalgia (FM) is a chronic pain syndrome with a complex, multifactorial and not completely known etiopathogenesis that affects mainly women. The prevalence of FM in studies carried out in the adult female population ranged between 2.4 and $6.8 \%$ [1]. FM is often associated with high levels of somatic symptoms (pain, fatigue, stiffness, and numbness), cognitive impairments, disturbances of sleep, and psychiatric comorbidity (psychological distress, mood

\footnotetext{
* Correspondence: gabho@hotmail.com

1Department of Rheumatology, Hospital General Regional número 1, Delegación Yucatán, Instituto Mexicano del Seguro Social, Calle 41 S/N, Colonia Industrial, 97150 Mérida, Yucatán, Mexico

Full list of author information is available at the end of the article
}

disorders, and personality traits). These symptoms, which may be part of FM or separate comorbid illnesses (e.g., migraine, irritable bowel syndrome, chronic fatigue syndrome, and major depression) have the greatest impact on FM patients' quality of life and often result in an impaired physical function that profoundly influences the patient's normal life and leisure activities [2-4]. Women with FM have been shown to be less physically active than healthy women, especially regarding physical activity of moderate to high intensity [5].

Most studies evaluating disability in FM compare working patients versus those who do not work. Between 34 and $77 \%$ of women with FM are reported to work [6].

(c) The Author(s). 2019 Open Access This article is distributed under the terms of the Creative Commons Attribution 4.0 International License (http://creativecommons.org/licenses/by/4.0/), which permits unrestricted use, distribution, and 
Previous studies have reported FM people's working ability is associated to different variables: pain intensity, Fibromyalgia Impact Questionnaire score, psychological factors (anxiety, depression, and coping strategies) and job associated factors [7-9]. From these variables, chronic pain and mood disorders are the most frequently reported. Pain intensity could predict physical impact or functionality degree [10]. Related to mood changes, it is known that musculoskeletal disorders become more disabling when they coexist with depression and/or anxiety, even when each of them could act independently [11]. Mood disorders contribute to a worsening of the global executive dysfunction suffered by FM patients and have a negative impact on their daily functioning [12]. Women with FM have a less psychological well-being than healthy controls and patients with rheumatoid arthritis [13]. Further, greater psychological well-being was associated with less disability and fatigue, but not pain in women with FM [13].

Disability is present in FM and constitutes an important problem for patients, physicians, and social insurance offices. Understanding the determinants of disability in fibromyalgia could contribute to the development of effective interventions. The focus of research on pain related disability has been shifted from a biomedical view to a holistic perspective in which in addition to biomedical also psychological factors have their influence [9]. Psychological factors play a major role in the onset, exacerbation or maintenance of FM symptoms in the majority of patients [14]. Especially anxiety and depression seem to have a substantial influence on the level of disability in fibromyalgia [15]. Alexithymia has been traditionally conceptualized as a personality trait involving a lack of emotional awareness, difficulties in identifying and communicating feelings and an externally oriented cognitive thinking style [16]. Alexithymia has been associated with clinical pain and other factors related to FM, such as general distress, higher levels of pain, depression, anxiety and somatosensory amplification [17], so it could predict disability.

Methods to measure disability vary from one country to another and influence results. There are many ways of assessing disability, for example by self-reported questionnaires, by clinical assessment, or by work status, where measures vary depending on the purpose of the investigation. Usually, disability evaluation in FM studies is comparing working versus non-working people. However, disability estimation by working situation or number of handicapped subjects depends on social benefits, laws, working rules of each country, as well as unemployment rates. Therefore, comparison among different populations becomes difficult.

Assessment of FM disability is complex and controversial, mostly due to the absence of objective evidence related to its severity, subjective manifestations, psychiatric comorbidities and differences in evaluation instruments. However, disability could be one of its main results. Patient-reported outcomes (PRO) become relevant for research due to this lack of anatomical, laboratory or physiological abnormalities, as they allow free symptoms' expression by the subject without observer bias [18]. Information about FM disability using self-reported questionnaires is limited.

The World Health Organization-Disablement Assessment Schedule (WHODAS 2.0) instrument is unique in its potential to determine the level of functioning of an individual irrespective of the type of disability or diagnosis $[19,20]$. One of the advantages of using the WHODAS 2.0 to measure disability is that it gives possibilities to compare different patient groups in further studies. The objectives of the present study were to evaluate self-reported disability in women with fibromyalgia and assess the relationship between pain intensity, depression, anxiety, alexithymia and disability in women with fibromyalgia.

\section{Methods \\ Study design}

This study consisted of a cross-sectional of consecutive patients with FM. All patients visiting the outpatient Department of Rheumatology of the Hospital General Regional 220, from May 2015 to December 2017 were asked to participate. Patients included recent and previously FM diagnosed subjects, invited to participate while they checked-in. Inclusion criteria were age $\geq 18$ years and fulfilled the preliminary criteria of the American College of Rheumatology [21]. Patients were excluded from the study if they were under age of 18 years or unable to give valid consent, presence of any acute disease that could modify the symptoms (fracture, trauma, fever and infection), coexistence of another rheumatic disease (for example, rheumatoid arthritis, systemic lupus erythematosus and ankylosing spondylitis), psychosis, suicide attempts in the last 3 months, and congenital or acquired disability known. Patients currently involved in compensation or litigation related to their disability were excluded. All participants were informed about the study procedures and gave their informed consent. The study was approved by the local research Ethic Committee (Project-Number R-2014-1503-88) and was conducted following the Helsinki Declaration. All participants signed an informed consent prior to their inclusion.

\section{Sample size}

Whitehead presents the sample size formulae for ordinal data [22]. To use Whitehead's formulae we need to specify an effect size (odds ratio ordinal). The odds ratio ordinal (OR) is the odds of a subject being in a given category or lower in one group compared with the odds 
in the other group. Because there are no previous studies that report effect size of the differences between the groups (depression vs. non-depression) by the disability degree of the WHODAS 2.0 in patients with fibromyalgia. Sample size was based on the results of a pilot study with 27 subjects. A sample size of 55 per group (depression vs. non-depression) was set, assuming $\alpha$ error of 0.05 , and $95 \%$ power to detect an effect size of 6.5 (OR ordinal $=6.5$ ).

\section{Measurement of variables}

Participants completed a printed survey with questions on demographic information (age, years of education, and disease duration) and self-reported questionnaires. Validated Spanish questionnaires were answered by patients themselves in a printed format, previous to medical consultation with no limitation in time to answer, they are described as follows:

Disability. The WHODAS 2.0 is a standardised measure developed by the World Health Organization (WHO) to assess the extent of activity limitation experienced by an individual, independent from a medical diagnosis. It evaluates 6 dimensions of individual functioning that define the disability construct included in the International Classification of Functioning (ICF) [20]. 36-item version was used, obtaining partial punctuations for each domain and providing a global functioning profile. The scores assigned to each item are recoded and summed in each domain with a range from 0 (best) to 100 (worst), using complex scoring [23]. In order to analyze the disability degree, thresholds based on ICF qualifying percentages were used [24]: absent (0-4.9), mild (5-24.9), moderate (2549.9), severe (50-95.9) and extreme (96-100).

Pain intensity was determined using the Visual Analogue Scale (VAS). VAS is a horizontal $10 \mathrm{~cm}$ line, where the beginning is pain absence $(0)$ and the end is the worst imaginable pain (10).

Alexithymia was evaluated using the Modified Toronto Alexithymia Scale (TAS-20) [25]. The scale's global score measures three different aspects of alexithymia: difficulty identifying feelings and distinguishing between feelings and bodily sensations, difficulty describing feelings, and externally oriented thinking. The cut-off points used to divide patients were those without alexithymia (global score $\leq 60$ ) and those with alexithymia (global score $\geq 61$ ).

The presence of depressive symptoms and anxiety were evaluated using the Hospital Anxiety and Depression Scale. It comprises 14 items in a range $0-3$, and it is divided into two subscales, one for depression and the other for anxiety. The cut-off point to classify subjects with clinically relevant symptomatology was eight in both subscales [26].

Patient drug compliance was determined using the Compliance Questionnaire Rheumatology (CQR). The global CQR score was then used to calculate a score from 0 to 100, where higher score implies greater adherence. The cut-off point of $80 \%$ was chosen to define adherence [27].

All patients underwent a protocol-based clinical evaluation by rheumatologist, including a physical examination, and routine laboratory studies (e.g., complete blood count and automated chemical analysis).

\section{Statistical analysis}

Data are shown as mean and standard deviation for normal distribution variables; median and interquartile range for non-normal distribution data. The chi-squared test is used to compare the distribution of a categorical variable. ANOVA test was used to evaluate the difference between mean values of pain intensity and disability degree; besides, Bonferroni post-hoc test was applied to know the different groups with an alpha value of 0.05 . Effect sizes were analyzed by computing the omegasquared coefficient, considering $0.01,0.06$, and 0.14 as threshold values to estimate low, medium, and large size effects, respectively [28]. Mann-Whitney $U$ test assessed the difference among score of WHODAS 2.0 in the presence of depression, anxiety and alexithymia. A size effect estimation was reported with a $\mathrm{z}$ statistic, considering $0.1,0.3$, and 0.5 as threshold values to estimate low, medium, and large size effects, respectively [28]. An ordinal logistic regression was performed to estimate the resulting ordinal variable (degree of disability), from a set of explanatory variables: age, years of education, body mass index, time since fibromyalgia diagnosis, pain intensity, depression, anxiety, alexithymia and work status. Predictors that were associated to disability degree by a predetermined $p$ value of 0.20 or less were selected and used in a multivariable ordinal logistic regression model, using stepwise forward selection. In proportional odds models, the outcome variable is ordered with multiple levels, and we estimate the odds of being at or below a particular category [29]. The proportional odds model assume that each predictor has the same effects across the categories of the ordinal outcome variable [29]. In order to evaluate its validity, supposed proportional risk was verified by Brant test. All $p<0.05$ values were considered statistically significant. Statistical analysis was performed with Stata-15 (2017, StataCorp, College Station, TX, USA).

\section{Results}

A total of 137 women with fibromyalgia were evaluated and 115 were included. Exclusion reasons were: 1 patient committed to the psychiatric service due to suicide ideation, 5 refused consent to participate or withdrew consent, 2 patients due to illiteracy, 1 for disability previous to fibromyalgia diagnosis, 11 patients due to coexistence 
of a rheumatic disease and 2 patients for incomplete questionnaire. The participants' age ranged from 31 to 66 years old (mean 48.4). Their median duration of symptoms 48 months and the mean of education level 10.1 years (Table 1). Fifty-seven (49.57\%) patients were in full or part-time jobs. Non-working women were 44.35\% $(n=51)$ housewives, $3.48 \%(n=4)$ unemployed and $2.61 \%(n=3)$ retired. Depressive disorder was observed in $63.4 \%$ of the subjects, anxiety disorder in $74.7 \%$ and alexithymia in $67.2 \%$ of them. All patients presented some degree of pain; mean pain intensity was $6.52 \mathrm{~cm}$ (minimum 1.4-maximum 10). Just one subject $(0.8 \%)$ was reported without disability (she was excluded from ANOVA and ordinal logistic regression analysis) and no cases were observed with extreme disability. The patient without disability was a 48-year-old woman with musculoskeletal pain for 48 months, pharmacological treatment with venlafaxine and good adherence. She presented pain of intensity $78 \mathrm{~mm}$ in the VAS, but without anxiety, depression or alexithymia.

Approximately $87 \%$ of the subjects $(n=100$, including the patient without disability) received pharmacological treatment. The average number of drugs prescribed per patient 2.1 with a range between 1 and 5 . There was not a significant difference between groups in the total number of drugs consumed. Treatments received for FM included: non-steroid anti-inflammatory drugs (NSAIDs) 21.9\%; celecoxib 30.7\%; Pregabalin or Gabapentin 52.6\%; Antidepressants $57.4 \%$ (add one patient without disability); Tramadol-acetaminophen 32.4\%; Benzodiazepines 9.6\%; and Quetiapine 1.7\% (Table 1). The result of the Compliance Questionnaire Rheumatology (CQR), including the patient without disability, was available in 87 patients (Table 1). Thirty-eight patients $(38 / 87 ; 43.7 \%)$ had a weighted CQR score of $\leq 80 \%$ (considered as "non-adherence") and 50 patients $(50 / 87 ; 56.3 \%)$ had a weighted CQR score of $>80 \%$ (considered as "good adherence").

Participants had moderate to severe levels of disability measured by the WHODAS 2.0, with a mean of $43.8 \pm$ 16.5 (95\%CI 40.7-46.9). WHODAS 2.0 domain analysis revealed that daily life activities, mobility and social participation were the most affected, and, self-care was the least affected (Table 2). Pain intensity mean scores for mild, moderate and severe disability were $5.0 \pm 2.3(95 \% \mathrm{CI}$ 3.5-6.4), $6.1 \pm 2.1$ (95\%CI 5.5-6.8) and $7.3 \pm 1.6$ (95\%CI 6.8-7.8), respectively (Fig. 1). Post hoc analysis revealed a statistically significative difference in pain intensity mean score for the conditions: mild versus severe disability $(p=$ $0.001)$, and moderate versus severe disability $(p=0.01)$. The difference between mild and moderate disability groups resulted not significative. The omega squared statistic $\left(\omega^{2}=0.12\right)$ indicted a medium effect size.

Table 1 Socio-demographic and clinical variables of the 114 patients with disability

\begin{tabular}{|c|c|c|c|c|c|c|}
\hline & Mild $(n=13)$ & Moderate $(n=54)$ & Severe $(n=47)$ & Total $(n=114)$ & $P$ value & Size effect \\
\hline Age in years & $48.2 \pm 5.9$ & $49.9 \pm 7.2$ & $46.8 \pm 6.4$ & $48.4 \pm 6.8$ & 0.07 & $0.02^{a}$ \\
\hline Years of education & $10 \pm 3.5$ & $10.2 \pm 3.6$ & $10.1 \pm 3.2$ & $10.1 \pm 3.4$ & 0.98 & $0.01^{\mathrm{a}}$ \\
\hline Time since onset of pain (months) & $48(24)$ & $48(64)$ & $36(42)$ & $48(60)$ & 0.22 & $0.02^{b}$ \\
\hline Time since FM diagnosis (months) & $36(46)$ & $34(36)$ & $12(36)$ & $24(42)$ & 0.08 & $0.03^{b}$ \\
\hline Body mass index & $29.1 \pm 4.5$ & $28.4 \pm 3.1$ & $28.9 \pm 3.7$ & $28.7 \pm 3.5$ & 0.72 & $0.01^{\mathrm{a}}$ \\
\hline Pain Intensity & $5.0 \pm 2.3$ & $6.2 \pm 2.1$ & $7.3 \pm 1.6$ & $6.5 \pm 2.1$ & $<0.001$ & $0.12^{\mathrm{a}}$ \\
\hline Number of drugs & $2.3 \pm 1.1$ & $2.12 \pm 1.2$ & $2.08 \pm 1.2$ & $2.1 \pm 1.2$ & 0.85 & $0.01^{\mathrm{a}}$ \\
\hline Pharmacological treatment & 11 [84.6] & 49 [90.7] & 39 [82.9] & 99 [86.8] & 0.49 & - \\
\hline Celecoxib & $5[38.4]$ & $21[38.8]$ & 9 [19.1] & $35[30.7]$ & 0.08 & \\
\hline Nonsteroidal anti-inflammatory drugs & $3[23.0]$ & 9 [16.6] & $13[27.6]$ & 25 [21.9] & 0.41 & \\
\hline Pregabalin or Gabapentin & $6[46.1]$ & 30 [55.5] & $24[51.0]$ & $60[52.6]$ & 0.79 & \\
\hline Antidepressants & 9 [69.2] & $27[50.0]$ & 29 [61.7] & $65[57.0]$ & 0.31 & \\
\hline Tramadol-acetaminophen & $5[38.4]$ & 19 [35.1] & $13[27.6]$ & 37 [32.4] & 0.64 & \\
\hline Benzodiazepines & $0[0]$ & $7[12.9]$ & $4[8.5]$ & $11[9.6]$ & 0.34 & \\
\hline Quetiapine & $0[0]$ & $0[0]$ & $2[4.2]$ & $2[1.7]$ & 0.23 & \\
\hline \multicolumn{7}{|l|}{ Medication adherence } \\
\hline Non-pharmacological treatment & $2[15.3]$ & $5[9.2]$ & $8[17.0]$ & 15 [13.0] & 0.35 & \\
\hline Adherent & $5[38.4]$ & $26[48.1]$ & $17[36.1]$ & $48[42.1]$ & & \\
\hline Non-adherent & $2[15-3]$ & 19 [35.1] & $17[36.1]$ & 38 [33.0] & & \\
\hline Unknown & $4[30.7]$ & $4[7.4]$ & $5[10.6]$ & 13 [11.3] & & \\
\hline
\end{tabular}

Data. Mean \pm Standard deviation; median (interquartile range); Frequency [percent]

Interpretation. ${ }^{a}$ Omega squared $=0.01-0.059$ is a low association. $0.06-0.13$ is a medium association. $\geq 0.14$ is a large association. ${ }^{b}$ Epsilon squared 
Table 2 Mean and median score for each domain and the global score of WHODAS 2.0

\begin{tabular}{llll}
\hline Domains & Mean \pm Standard deviation & Median (25th percentile-75th percentile) & Minimum - maximum score \\
\hline Lifeactivities (Household) & $54.8 \pm 24.0$ & $50(40-70)$ & $0-100$ \\
Mobility & $50.0 \pm 20.4$ & $50(37.5-68.7)$ & $6.2-100$ \\
Participation & $48.2 \pm 19.0$ & $45.8(37.5-62.5)$ & $8.3-87.5$ \\
Life activities (School/Work) & $43.7 \pm 21.0$ & $42.8(28.5-57.1)$ & $0-85.7$ \\
Cognition & $40.8 \pm 19.5$ & $40(30-55)$ & $0-90$ \\
Getting along & $35.3 \pm 26.8$ & $33.3(8.3-50)$ & $0-100$ \\
Self-care & $28.6 \pm 23.2$ & $30(10-50)$ & $0-90$ \\
Global score & $43.8 \pm 16.5$ & $43.4(31.5-55.4)$ & $4.3-81-5$ \\
\hline
\end{tabular}

The depression group scored significantly higher than the non-depression group on the global WHODAS 2.0 score (50 vs $36.4, p<0.001$ ). Similarly, the anxiety group scored significantly higher than the non-anxiety group (46.1 vs $33.6, p=0.007$ ), and alexithymic group scored significantly higher than the non-alexithymic group (50 vs 33.6, $p<0.001$ ) (Fig. 1). Effect size was medium association for depression (0.33) and alexithymia (0.38), and low association for anxiety (0.25).

The odds ratio (OR) obtained from ordinal logistic regression models for different variables represent disability in the index group compared with that in the reference group. Ordinal logistic regression analysis, revealed a significant association among presence of depression (OR 2.72, 95\% CI 1.28-5.75, $p=$ 0.009 ), anxiety (OR $2.49,95 \% \mathrm{CI} 1.09-5.68, p=0.029$ ), pain intensity $(1.44,95 \% \mathrm{CI} 1.19-1.74, p<0.001)$ and alexithymia (OR 4.26, 95\%CI 1.80-10.08, $p<0.001$ ) with disability degree. Variables as age and working situation (working versus non-working) showed a $p$ value between 0.05 and 0.20 , thus, they were considered for multivariate analysis. The main explanatory variables of disability degree were pain intensity and alexithymia (Table 3 ). Table 4 shows associations between different variables and disability in domainspecific scores. Pain intensity explained the global disability degree and its domains except for the cognitive
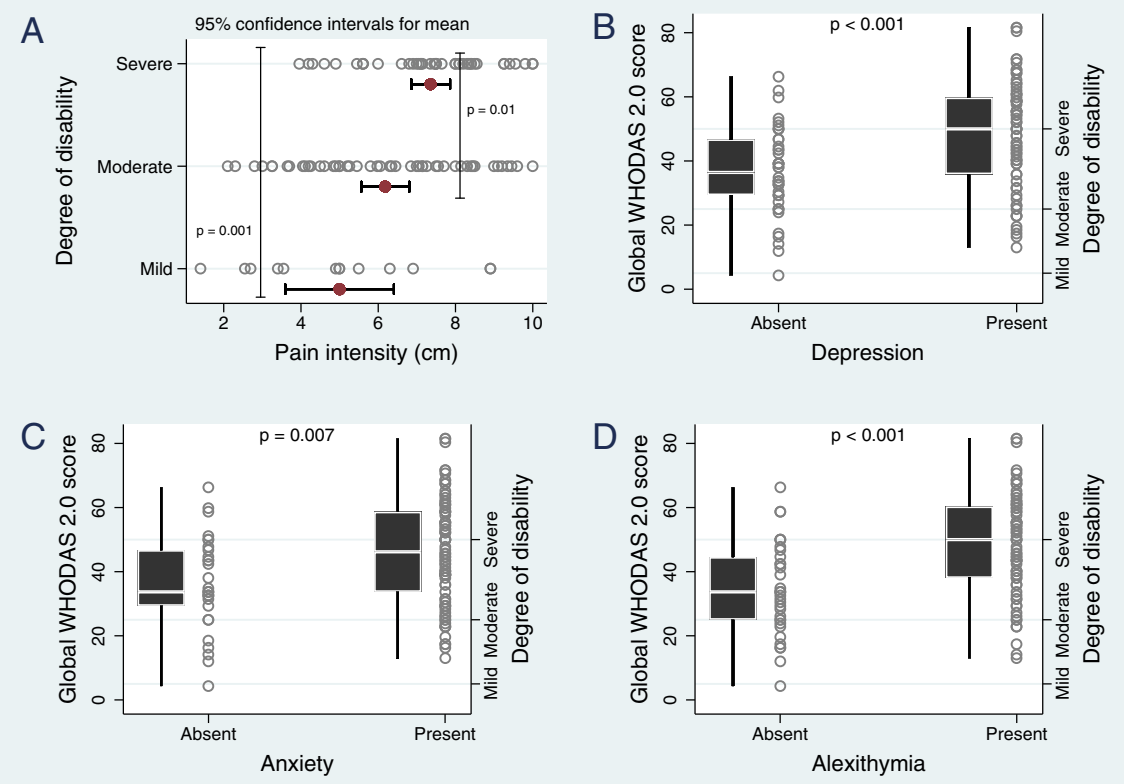

Fig. 1 Graph of 95\% confidence intervals of mean pain intensity by disability degree (a). Box plot of WHODAS 2.0 global score by depression (b) anxiety (c) and alexithymia (d). Upper and lower whiskers represent 1.5 times and - 1.5 times interquartile range; upper and lower hinges represent 25 and $75 \%$ quartiles; middle represents median or $50 \%$ quartile. Each dot represents a value 
Table 3 Adjusted Odds Ratio of predictors of disability degree in Fibromyalgia women

\begin{tabular}{|c|c|c|c|c|c|c|c|}
\hline \multirow[b]{2}{*}{ Model 1} & \multirow{2}{*}{$\begin{array}{l}\text { Explanatory variables } \\
\text { Alexithymia }\end{array}$} & \multirow{2}{*}{$\begin{array}{l}\text { Adjusted Odds } \\
\text { Ratio (OR) } \\
4.02\end{array}$} & \multirow{2}{*}{$\begin{array}{l}P \text { value } \\
0.003\end{array}$} & \multicolumn{2}{|c|}{ 95\% Confidence Interval } & \multirow{2}{*}{$\begin{array}{c}\text { Pseudo } R^{2} \\
0.13\end{array}$} & \multirow{2}{*}{$\frac{\text { Brand test }}{0.79}$} \\
\hline & & & & 1.60 & 10.05 & & \\
\hline & Pain Intensity & 1.42 & 0.001 & 1.16 & 1.74 & & \\
\hline \multirow[t]{2}{*}{ Model 2} & Alexithymia & 3.58 & 0.004 & 1.49 & 8.64 & 0.07 & 0.30 \\
\hline & Depression & 2.15 & 0.066 & 0.95 & 4.87 & & \\
\hline \multirow[t]{2}{*}{ Model 3} & Alexithymia & 3.96 & 0.005 & 1.66 & 9.44 & 0.06 & 0.41 \\
\hline & Anxiety & 1.66 & 0.25 & 0.69 & 3.98 & & \\
\hline \multirow[t]{3}{*}{ Model 4} & Alexithymia & 3.71 & 0.006 & 1.46 & 9.39 & 0.13 & 0.42 \\
\hline & Pain Intensity & 1.40 & 0.001 & 1.14 & 1.72 & & \\
\hline & Depression & 1.61 & 0.27 & 0.68 & 3.81 & & \\
\hline \multirow[t]{5}{*}{ Model 5} & Alexithymia & 3.28 & 0.014 & 1.27 & 8.46 & 0.15 & 0.11 \\
\hline & Pain Intensity & 1.39 & 0.001 & 1.13 & 1.71 & & \\
\hline & Depression & 1.76 & 0.20 & 0.73 & 4.28 & & \\
\hline & Work status & 1.99 & 0.11 & 0.85 & 4.64 & & \\
\hline & Age in years & 0.97 & 0.52 & 0.92 & 1.04 & & \\
\hline
\end{tabular}

Pain intensity in centimeter (0-10). Reference category (exposure absent). Depression $(0=$ absent $/ 1=$ present $)$, Alexithymia $(0=a b s e n t / 1=$ present $)$, Work Status $(0=\text { Not worker, } 1=\text { Worker })^{*}$

A non-significant $(p>0.05)$ Brant test indicates that the proportional odds assumption is not violated

one. Depression explained cognitive and personal relation domains. Neither age, anxiety nor employment status had a significant influence in the model. On the other hand, alexithymia explained global disability degree and all domains of WHODAS 2.0 questionnaire.

\section{Discussion}

The majority of our patients reported a significant impact on functionality. Results revealed that most of the patients with fibromyalgia perceived themselves with moderate to severe disability. Overall, the degree of

Table 4 Association between WHODAS disability domains and Pain Intensity, Depression and Alexithymia

\begin{tabular}{|c|c|c|c|c|c|}
\hline Dependent variable & Predictor variables & Odds Ratio & 95\% Confidence Interval & Standard error & $P$ value \\
\hline \multirow[t]{3}{*}{ Cognition } & Alexithymia & 3.26 & $1.36-7,83$ & 1.45 & 0.008 \\
\hline & Depression & 2.85 & $1.22-6.66$ & 1.23 & 0.015 \\
\hline & Pain intensity & 1.06 & $0.89-1.27$ & 0.09 & 0.47 \\
\hline \multirow[t]{3}{*}{ Mobility } & Pain intensity & 1.35 & $1.09-1.66$ & 0.14 & 0.004 \\
\hline & Alexithymia & 3.26 & $1.36-7.83$ & 1.45 & 0.008 \\
\hline & Depression & 1.10 & $0.46-2.60$ & 0.48 & 0.82 \\
\hline \multirow[t]{3}{*}{ Self-care } & Pain intensity & 1.36 & $1.13-1.64$ & 0.12 & 0.001 \\
\hline & Alexithymia & 2.78 & $1.23-6.29$ & 1.15 & 0.014 \\
\hline & Depression & 1.40 & $0.64-3.07$ & 0.56 & 0.39 \\
\hline \multirow[t]{3}{*}{ Getting along } & Depression & 2.57 & $1.16-5.69$ & 1.12 & 0.020 \\
\hline & Alexithymia & 2.64 & $1.14-6.08$ & 1.04 & 0.023 \\
\hline & Pain intensity & 1.04 & $0.87-1.24$ & 0.09 & 0.60 \\
\hline \multirow[t]{3}{*}{ Life activities (Household) } & Pain intensity & 1.22 & $1.00-1.49$ & 0.12 & 0.041 \\
\hline & Alexithymia & 3.04 & $1.25-7.40$ & 1.38 & 0.014 \\
\hline & Depression & 1.45 & $0.60-3.48$ & 0.64 & 0.39 \\
\hline \multirow[t]{3}{*}{ Participation } & Pain intensity & 1.26 & $1.04-1.53$ & 0.12 & 0.018 \\
\hline & Alexithymia & 2.87 & $1.18-6.96$ & 1.29 & 0.019 \\
\hline & Depression & 1.97 & $0.84-4.62$ & 0.85 & 0.11 \\
\hline
\end{tabular}

Pain intensity in centimeter $(0-10)$, depression $(0=$ abscent $/ 1=$ present $)$, Alexithymia $(0=$ abscent $/ 1=$ present $)$ 
disability in this sample of patients with FM was 1.5 to 2 times greater than that reported in comparable studies of patients with other rheumatologic conditions such as osteoarthritis [30], systemic lupus erythematosus [31, 32], and ankylosing spondylitis [31]. Besides, degree of disability was similar to rheumatoid arthritis patients [31, 33, 34].

WHODAS 2.0 mean score found in the present study was lower than the reported by Casanueva-Fernández et al. [18]. Even when similarities were found in the most affected domains (daily life activities and mobility). Thus, revealing that symptoms of FM syndrome affect mostly daily life functioning in people even without working disputes. The disability assessment process itself and litigation for financial compensation can act as aggravating factors, by influencing reports of symptom severity [35-37]. Like in other studies [6,38], about $50 \%$ of the patients were working women. In contrast to reports in developed countries that point out that one third of fibromyalgia workers receive disability benefits [8]; in our country, these supports, paid by healthcare system or legal issues, are still unusual. Some authors suggest that disability is based on patient motivation and financial compensation $[39,40]$. Patients tend to exaggerate their symptoms, because this increases their chances of receiving financial compensation [41-44]. However, one study could not confirm this association [45].

The level of self-reported disability in patients with fibromyalgia seemed best explained by their pain intensity and alexithymia. The pain intensity of FM is in itself disabling. The present results agree with previous reports in which pain intensity is a predictor of physical impact or functionality degree in FM subjects [10]. Pain is both a symptom and a contributor to other symptoms. Severe pain creates fatigue, impairs concentration, negative mood, and diminishes overall activity level [46]. Chronic pain could cause changes in functioning and represent an important disability in any effort done in the past with no difficulties. Pain interferes with normal achievement of several activities including movement, leisure activities, sleep, self-care, housework, job and psychological functioning [47]. A hypothesis about how pain intensity produces functional disability is that a reduction in work ability might be due to a decrease in the ability to activate motor units completely, thus, lessening muscular strength and resistance. The pain influences the ability to fully activate the muscles, resulting in reduced muscular strength and endurance [48].

Psychological factors seem to play a pronounced disabling role in fibromyalgia [9]. Several psychological conditions, such as depression, anxiety, and stress, have been associated with disability [9, 13, 49-52]. A relevant finding of the present report is that alexithymia was found to be a predictor of the degree of global disability and in all domains. Scarce information is available about the relationship between disability and alexithymia. A study demonstrated [53] that people with alexithymia showed higher disability scores due to pain, as assessed by the Pain Disability Scale. Alexithymia was not an independent predictor, since depression was a complete mediator between the TAS-20 score and the Pain Disability Scale [53]. Despite this result, when using a generic instrument to assess disability, alexithymia was significantly associated with disability.

Alexithymia's exact role in FM pathology is still unknown, it has been proposed that alexithymia interferes with the successful self-regulation of negative emotions, resulting in increased negative affect and chronic sympathetic hyperarousal, which may contribute to the development or exacerbation of somatic disease and pain [54].

People with FM are at increased risk for depression. Both depression and having FM have an independent, negative effect on physical function [55]. Associations found between disability and depression are less consistent than the ones between pain intensity and disability, some studies have reported depression as independent predictor of disability [55], while others have shown that this effect is lost when adjusting for covariates. In the present study, no significative association was revealed between depression symptoms and disability degree. However, depression was a predictor of cognitive disability and personal relations, similar to the reported by Silva et al. [56], who revealed depression as the most important predictor for cognition, personal relations and social participation in patients with musculoskeletal pain. The influence of mood symptoms in subjective ratings of cognitive functioning has also been reported in patients with FM [12]. In contrast, Arnstein et al. [57] reported that depression was not a significative predictor of disability, after adjusting the effect by pain intensity and self-efficacy beliefs.

Based on our results, patients with fibromyalgia and disability might benefit from psychological interventions that directly target emotional awareness processes, such as cognitive-behavioral therapy (CBT) and MindfulnessBased Therapies (MBT) [58-60]. CBT focuses on coping strategies, emotional control and cognitive psychology and has shown successful results in counteracting mood disorders and disability in FM patients [61]. FM patients treated with MBT have reported pain, sleep or psychological distress improvement [62].

Some limitations of the present study should be taken into account in future research. First, because our patients were drawn from a referral clinic, they may represent the most severely afflicted patients and hence not be representative of most patients with FM. The results are not generalizable beyond women being treated in 
rheumatology practices. Second, the cross-sectional design does not allow to establish causal relationships. Third, given the likely bidirectional nature of the relationship between pain, depression and alexithymia. Psychological distress or mood disorders might mediate the links between alexithymia and pain. Unfortunately, cross-sectional analyses cannot differentiate these two possibilities. Fourth, we have no way of directly validating the self-reported disability in this study. Finally, our results could not be extrapolated to determine disability in the medico-legal context. In the context of working disability, self-report instruments are not considered as an objective measure of the disability degree since there is a secondary profit and information is frequently manipulated, particularly exaggerating the symptoms, not only in FM subjects [63]. The present study serves only to identify some factors that may affect self-reported disability.

Our results support that FM is associated with substantial self-reported disability. Disability evaluation by self-report measures in FM could be the best method for its characterization and the most adequate to understand subjacent mechanisms of disability as they reflect values and priorities of the patients. WHODAS 2.0 was designed to record and measure patient's opinions about their disability experiences, either if disability is due to a mental and/or physical deficiency [63].

\section{Conclusions}

Most of the patients with fibromyalgia (tertiary care setting) perceived themselves with moderate to severe disability. The main explanatory variables of the perceived disability were the pain intensity and psychological factors (alexithymia and depression). The present study can help enhance the knowledge of disability in FM by including the patient perspective. Future longitudinal work may offer a better understanding of the causal direction of pain, alexithymia, mood disorders and disability.

\section{Acknowledgements}

Not applicable.

\section{Authors' contributions}

All authors contributed equally to write and review the manuscript. Both authors read and approved the final manuscript.

\section{Funding}

This study did not receive funding or financial support.

\section{Availability of data and materials}

The datasets used and/or analysed during the current study are available from the corresponding author on reasonable request.

\section{Ethics approval and consent to participate}

This study was approved by the local research ethic committee of the Instituto Mexicano del Seguro Social. All participants signed an informed consent prior to their inclusion.
Consent for publication

Not applicable.

\section{Competing interests}

The authors declare that they have no competing interests.

\section{Author details}

'Department of Rheumatology, Hospital General Regional número 1, Delegación Yucatán, Instituto Mexicano del Seguro Social, Calle 41 S/N, Colonia Industrial, 97150 Mérida, Yucatán, Mexico. ${ }^{2}$ Centro de Investigación en Ciencias de la Salud, Campus Norte Huixquilucan, Universidad Anáhuac México, Ciudad de México, Mexico.

Received: 4 July 2019 Accepted: 10 September 2019

Published online: 23 October 2019

\section{References}

1. Marques AP, Santo A, Berssaneti AA, Matsutani LA, Yuan SLK. Prevalence of fibromyalgia: literature review update. Rev Bras Reumatol Engl Ed. 2017;57:356-63.

2. Kleinman N, Harnett J, Melkonian A, Lynch W, Kaplan-Machlis B, Silverman SL. Burden of fibromyalgia and comparisons with osteoarthritis in the workforce. J Occup Environ Med. 2009:51:1384-93.

3. Henriksson C, Burckhardt C. Impact of fibromyalgia on everyday life: a study of women in the USA and Sweden. Disabil Rehabil. 1996;18:241-8.

4. Palstam A, Mannerkorpi K. Work ability in fibromyalgia: an update in the 21st century. Curr Rheumatol Rev. 2017;13:180-7.

5. McLoughlin MJ, Colbert LH, Stegner AJ, Cook DB. Are women with fibromyalgia less physically active than healthy women? Med Sci Sports Exerc. 2011;43:905-12.

6. Mannerkorpi K, Gard G. Hinders for continued work among persons with fibromyalgia. BMC Musculoskelet Disord. 2012;13:96.

7. Howard KJ, Mayer TG, Neblett R, Perez Y, Cohen H, Gatchel RJ. Fibromyalgia syndrome in chronic disabling occupational musculoskeletal disorders: prevalence, risk factors, and posttreatment outcomes. J Occup Environ Med. 2010;52:1186-91.

8. Fitzcharles MA, Ste-Marie PA, Rampakakis E, Sampalis JS, Shir Y. Disability in fibromyalgia associates with symptom severity and occupation characteristics. J Rheumatol. 2016;43:931-6.

9. Verbunt JA, Pernot DH, Smeets RJ. Disability and quality of life in patients with fibromyalgia. Health Qual Life Outcomes. 2008;6:8.

10. White KP, Speechley M, Harth M, Ostbye T. Comparing self-reported function and work disability in 100 community cases of fibromyalgia syndrome versus controls in London, Ontario: the London fibromyalgia epidemiology study. Arthritis Rheum. 1999;42:76-83.

11. Bair MJ, Wu J, Damush TM, Sutherland JM, Kroenke K. Association of depression and anxiety alone and in combination with chronic musculoskeletal pain in primary care patients. Psychosom Med. 2008; 70:890-7.

12. Gelonch O, Garolera M, Valls J, Rossello L, Pifarre J. Executive function in fibromyalgia: comparing subjective and objective measures. Compr Psychiatry. 2016;66:113-22.

13. Schleicher H, Alonso C, Shirtcliff EA, Muller D, Loevinger BL, Coe CL. In the face of pain: the relationship between psychological well-being and disability in women with fibromyalgia. Psychother Psychosom. 2005;74:231-9.

14. Perrot S. Fibromyalgia a misconnection in a multi-connected world? Eur J Pain. 2019;23(5):866-73.

15. van Houdenhove B, Neerinck E, Onghena P, Lysens R, Vertommen H. Premorbid "overactive" lifestyle in chronic fatigue syndrome and fibromyalgia :an etiological factor or proof of good citizenship? J Psychosom Res. 2001;51:571-6.

16. Montoro $\mathrm{Cl}$, Reyes del PAso GA, Duschek S. Alexithymia in fibromyalgia syndrome. Pers Individ Dif. 2016;102:170-9.

17. Di Tella M, Ghiggia A, Tesio V, Romeo A, Colonna F, Fusaro E, et al. Pain experience in fibromyalgia syndrome: the role of alexithymia and psychological distress. J Affect Disord. 2017;208:87-93.

18. Casanueva-Fernández B, Sánchez-Villar R, Pérez-Martín A, Rodero-Fernández B, Belenguer-Prieto R, González-Gay MA, et al. Evaluación genérica y específica de discapacidad en pacientes con fibromialgia. Reumatol Clin. 2010;6:164 (Espec Cong). 
19. The world health organization. World health organization disablement assessment schedule ii, who-das ii. Geneva: World Health Organization; 2001.

20. Ustun TB, Chatterji S, Kostanjsek N, Rehm J, Kennedy C, Epping-Jordan J, et al. Developing the world health organization disability assessment schedule 2.0. Bull World Health Organ. 2010;88:815-23.

21. Wolfe F, Clauw DJ, Fitzcharles MA, Goldenberg DL, Katz RS, Mease P, et al. The american college of rheumatology preliminary diagnostic criteria for fibromyalgia and measurement of symptom severity. Arthritis Care Res (Hoboken). 2010;62:600-10.

22. Whitehead J. Sample size calculations for ordered categorical data. Stat Med. 1993;12:2257-71.

23. World health organization. (2014). Who disability assessment schedule 2.0 (whodas 2.0). Http://www.Who.Int/classifications/icf/whodasii/en/. Accessed 8 Jan 2016.

24. Ayuso-Mateos JL, Nieto-Moreno M, Sánchez-Moreno J, Vázquez-Barquero JL. Clasificación internacional del funcionamiento, la discapacidad y la salud (cif): Aplicabilidad y utilidad en la práctica clínica. Med Clin (Barc). 2006;126:461-4.

25. Pérez-Rincón H, Cortés J, Ortíz S, Peña J, Ruíz J, Diaz-Martínez A. Validación y estandarización de la versión española de la escala modificada de alexitimia de toronto. Salud Ment. 1997;20:30-4.

26. Lopez-Alvarenga J, Vazquez-Velazquez V, Arcila-Martinez D, Sierra-Ovando A, Gonzalez-Barranco J, Salin-Pascual R. Exactitud y utilidad diagnostica del hospital anxiety and depression scale (had) en una muestra de sujetos obesos mexicanos. Rev Investig Clin. 2002;54:403-9.

27. Arturi P, Schneeberger EE, Sommerfleck F, Buschiazzo E, Ledesma C, Maldonado Cocco JA, et al. Adherence to treatment in patients with ankylosing spondylitis. Clin Rheumatol. 2013;32:1007-15.

28. Fritz CO, Morris PE, Richler JJ. Effect size estimates: current use, calculations, and interpretation. J Exp Psychol Gen. 2012;141:2-18.

29. Liu X. Applied ordinal logistic regression using stata. California: SAGE Publications; 2016.

30. Silva C, Coleta I, Silva AG, Amaro A, Alvarelhao J, Queiros A, et al. Adaptation and validation of whodas 2.0 in patients with musculoskeletal pain. Rev Saude Publica. 2013;47:752-8

31. Ramos-Remus C, Castillo-Ortiz JD, Sandoval-Castro C, Paez-Agraz F, Sanchez-Ortiz A, Aceves-Avila FJ. Divergent perceptions in health-related quality of life between family members and patients with rheumatoid arthritis, systemic lupus erythematosus, and ankylosing spondylitis. Rheumatol Int. 2014;34:1743-9.

32. Kutlay S, Kucukdeveci AA, Elhan AH, Oztuna D, Koc N, Tennant A. Validation of the world health organization disability assessment schedule ii (whodasii) in patients with osteoarthritis. Rheumatol Int. 2011;31:339-46.

33. Baron M, Schieir O, Hudson M, Steele R, Kolahi S, Berkson L, et al. The clinimetric properties of the world health organization disability assessment schedule ii in early inflammatory arthritis. Arthritis Rheum. 2008;59:382-90.

34. Meesters JJ, Verhoef J, Liem IS, Putter H, Vliet Vlieland TP. Validity and responsiveness of the world health organization disability assessment schedule ii to assess disability in rheumatoid arthritis patients. Rheumatology. 2010;49:326-33

35. Gervais RO, Russell AS, Green P, Allen LM 3rd, Ferrari R, Pieschl SD. Effort testing in patients with fibromyalgia and disability incentives. J Rheumatol. 2001;28:1892-9.

36. Helfenstein M Jr, Goldenfum MA, Siena CA. Fibromyalgia: clinical and occupational aspects. Rev Assoc Med Bras. 2012:58:358-65.

37. Harris IA, Murgatroyd DF, Cameron ID, Young JM, Solomon MJ. The effect of compensation on health care utilisation in a trauma cohort. Med J Aust. 2009;190:619-22

38. Al-Allaf AW. Work disability and health system utilization in patients with fibromyalgia syndrome. J Clin Rheumatol. 2007;13:199-201.

39. Bennett RM. Fibromyalgia and the disability dilema. Arthritis Rheum. 1996; 39:1627-34.

40. Aarón LA, Alancon GS. Perceived physical and emotional trauma and precipitating events in fibromyalgia. Arthritis Rheum. 1997;40:453-60.

41. Blasco Claros L, Mallo Cano M, Mencia Presa A, Franch Barcelo J, Casaus Sataman P, Pena Roca J, et al. Clinical profiles in fibromyalgia patients of the community mental health center: a predictive index of psychopathological severity. Actas Esp Psiquiatr. 2006;34:112-22.

42. Pérez-Pareja J, Sesé A, González-Ordi H, Palmer A. Fibromyalgia and chronic pain: are there discriminating patterns by using the Minnesota multiphasic personality inventory-2 (mmpi-2)? Int J Clin Health Psychol. 2010;10:41-56.

43. Yunus MB, Ahles TA, Aldag JC, Masi AT. Relationship of clinical features with psychological status in primary fibromyalgia. Arthritis Rheum. 1991;34:15-21.
44. Capilla-Ramírez P, González-Ordi H, Santamaría P, Pérez-Nieto MA, Casado-Morales M. Fibromialgia: jexageración o simulación? Clinica y Salud. 2013;24:185-95.

45. Turk DC, Okifuji A, Starz TW, Sinclair JD. Effects of type of symptom onset on psychological distress and disability in fibromyalgia syndrome patients. Pain. 1996;68:423-30.

46. Turk DC, Dworkin RH. What should be the core outcomes in chronic pain clinical trials? Arthritis Res Ther. 2004;6:151-4.

47. Goncalves Silva A, Queirós A, Pacheco-Rocha N. Generic self-reported and performance based instruments: how to capture pain associated disability. Rev Port Saude Pública. 2016;34:125-33.

48. Henriksson C, Liedberg GM. Factors of importance for work disability in women with fibromyalgia. J Rheumatol. 2000;27:1271-6.

49. Henriksson CM, Liedberg GM, Gerdle B. Women with fibromyalgia: work and rehabilitation. Disabil Rehabil. 2005;27:685-94.

50. Ledingham J, Doherty S, Doherty M. Primary fibromyalgia syndrome--an outcome study. Br J Rheumatol. 1993;32:139-42.

51. Kurtze N, Gundersen KT, Svebak S. Quality of life, functional disability and lifestyle among subgroups of fibromyalgia patients: the significance of anxiety and depression. Br J Med Psychol. 1999;72 ( Pt 4:471-84.

52. Hauser W, Sarzi-Puttini P, Fitzcharles MA. Fibromyalgia syndrome: Under-, over- and misdiagnosis. Clin Exp Rheumatol. 2019;37(Suppl 116):90-7.

53. Saariaho AS, Saariaho TH, Mattila AK, Karukivi MR, Joukamaa MI. Alexithymia and depression in a chronic pain patient sample. Gen Hosp Psychiatry. 2013:35:239-45.

54. Huber A, Suman AL, Biasi G, Carli G. Alexithymia in fibromyalgia syndrome: associations with ongoing pain, experimental pain sensitivity and illness behavior. J Psychosom Res. 2009;66:425-33.

55. Kassam A, Patten SB. Major depression, fibromyalgia and labour force participation: a population-based cross-sectional study. BMC Musculoskelet Disord. 2006;7:4.

56. Silva AG, Alvarelhao J, Queiros A, Rocha NP. Pain intensity is associated with self-reported disability for several domains of life in a sample of patients with musculoskeletal pain aged 50 or more. Disabil Health J. 2013;6:369-76.

57. Arnstein $P$. The mediation of disability by self efficacy in different samples of chronic pain patients. Disabil Rehabil. 2000;22:794-801

58. Penacoba Puente C, Velasco Furlong L, Ecija Gallardo C, Cigaran Mendez M, McKenney K. Anxiety, depression and alexithymia in fibromyalgia: are there any differences according to age? J Women Aging. 2013;25:305-20.

59. Martinez MP, Sanchez Al, Miro E, Lami MJ, Prados G, Morales A. Relationships between physical symptoms, emotional distress, and pain appraisal in fibromyalgia: the moderator effect of alexithymia. J Psychol. 2015;149:115-40.

60. Calsius J, Courtois I, Stiers J, De Bie J. How do fibromyalgia patients with alexithymia experience their body? A qualitative approach. SAGE Open. 2015;5:1-10.

61. Atzeni F, Talotta R, Masala IF, Giacomelli C, Conversano C, Nucera V, et al. One year in review 2019: fibromyalgia. Clin Exp Rheumatol. 2019;37(Suppl 116):3-10.

62. Castelli L, Tesio V. Commentary: mindfulness training for reducing anger, anxiety, and depression in fibromyalgia patients. Front Psychol. 2016;7:740

63. Gold LH. Dsm-5 and the assessment of functioning: the world health organization disability assessment schedule 2.0 (whodas 2.0). J Am Acad Psychiatry Law. 2014;42:173-81.

\section{Publisher's Note}

Springer Nature remains neutral with regard to jurisdictional claims in published maps and institutional affiliations.

Ready to submit your research? Choose BMC and benefit from

- fast, convenient online submission

- thorough peer review by experienced researchers in your field

- rapid publication on acceptance

- support for research data, including large and complex data types

- gold Open Access which fosters wider collaboration and increased citations

- maximum visibility for your research: over $100 \mathrm{M}$ website views per year

At BMC, research is always in progress.

Learn more biomedcentral.com/submissions 